\title{
Remote Detection of Photoplethysmographic Systolic and Diastolic Peaks Using a Digital Camera
}

\author{
Daniel McDuff*, Student Member, IEEE, Sarah Gontarek, and Rosalind W. Picard, Fellow, IEEE
}

\begin{abstract}
We present a new method for measuring photoplethysmogram (PPG) signals remotely using ambient light and a digital camera that allows for accurate recovery of the waveform morphology (from a distance of $3 \mathrm{~m}$ ). In particular, we show that the peak-to-peak time between the systolic peak and diastolic peak/inflection can be automatically recovered using the second order derivative of the remotely measured waveform. We compare measurements from the face with those captured using a contact finger-tip sensor and show high agreement in peak and interval timings. Furthermore, we show that results can be significantly improved using orange, green and cyan color channels compared to the tradition red, green and blue channel combination. The absolute error in inter-beat-intervals was $26 \mathrm{~ms}$ and the absolute error in mean systolic-diastolic peak-to-peak times was $12 \mathrm{~ms}$. The mean systolic-diastolic peak-to-peak times measured using the contact sensor and the camera were highly correlated, $\rho=0.94(p<0.001)$. The results were obtained with a camera frame-rate of only $30 \mathrm{~Hz}$. This technology has significant potential for advancing healthcare.
\end{abstract}

\section{INTRODUCTION}

Photoplethysmography (PPG) is a low-cost and non-invasive method of measuring the cardiovascular blood volume pulse (BVP) via light transmitted through, or reflected from, the human body [1]. There are many clinical applications for PPG and it can reveal significant information about health and risk of cardiovascular diseases [2].

The peripheral pulse wave, as detected via PPG, characteristically exhibits systolic and diastolic peaks. The systolic peak is a result of the direct pressure wave traveling from the left ventricle to the periphery of the body, the diastolic peak (or inflection) is a result of reflections of the pressure wave by arteries of the lower body [3]. Figure 1 shows an example of a pulse waveform (black line) with the systolic and diastolic peaks labeled. The systolic-diastolic peak-to-peak time (SDPPT) is defined as the time between successive systolic and diastolic peaks/inflections.

Takazawa [4] evaluated the second derivative of the PPG waveform and found that it had a characteristic shape that aids in its interpretation. We use this approach to reveal diastolic inflections in cases were there is no observable diastolic peak within the BVP waveform. We compare the PPG waveforms and second order derivatives measured using a contact sensor

D. J. McDuff is with the Media Laboratory, Massachusetts Institute of Technology, Cambridge, MA 02139 USA. (e-mail: djmcduff@mit.edu).

S. Gontarek is with the Media Laboratory, Massachusetts Institute of Technology, Cambridge, MA 02139 USA (e-mail: sgontare@mit.edu).

R. W. Picard is a professor at the Media Laboratory, Massachusetts Institute of Technology, Cambridge, MA 02139 USA. (phone: 617-253-0611; e-mail: picard@media.mit.edu).

Copyright (c) 2013 IEEE. Personal use of this material is permitted. However, permission to use this material for any other purposes must be obtained from the IEEE by sending an email to pubs-permissions@ieee.org.

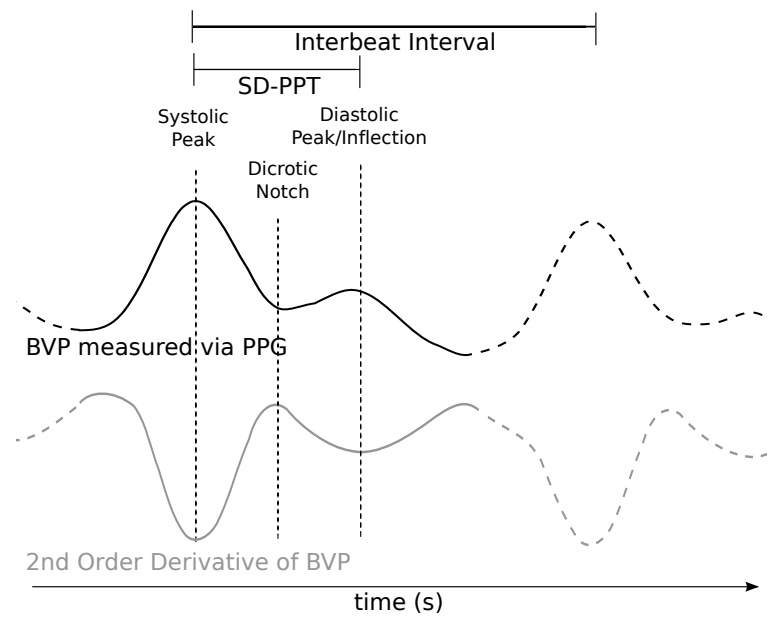

Fig. 1. Systolic-diastolic peak-to-peak time (SD-PPT) is calculated as the time between the systolic and diastolic peaks within the PPG signal. The inter-beat interval (IBI) is calculated as the time between systolic peaks. Calculating the second derivative of the PPG waveform can be highly beneficial in recovering the locations of the peaks.

and a digital camera. Figure 1 shows an example of the typical second order derivative shape of a BVP waveform (grey line).

Traditionally, PPG measurements have been captured using a contact sensor with dedicated light source. However, recent work has shown that heart rate (HR), respiration rate (RR) and heart rate variability (HRV) parameters can be extracted from PPG signals measured using a digital camera [5], [6], [7]. Motion compensation is possible when motion artifacts are problematic [8]. However, beyond accurate beat detection it has not been clear whether the morphology of the PPG signal could be accurately captured using such approaches. In this paper we show that accurate waveform morphology can be recovered automatically from the human face using ambient light and a digital camera at a distance of $3 \mathrm{~m}$.

The main contribution of this paper is to present an automated algorithm for extracting the BVP waveform and systolic and diastolic peaks from videos of the human face and show that these have a high degree of agreement with contact sensor measurements. In the remainder of the paper we will explain the automated approach, data collection, validation experiments and the results.

\section{METHODS}

\section{A. Extraction of the BVP from Video}

We use an automated method for recovering the BVP waveform from the recorded videos similar to that presented by McDuff et al. [7]. Figure 2(b) shows an overview of the 

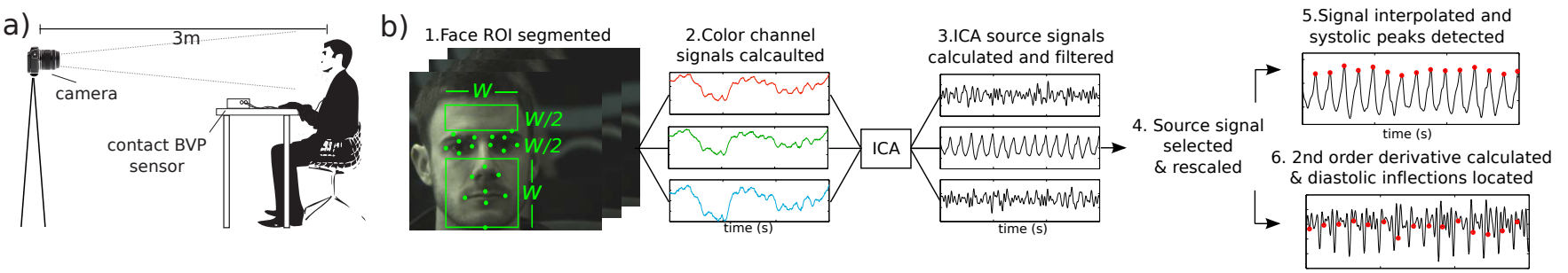

Fig. 2. a) Experimental set-up. Contact measurements of the blood volume pulse were collected using a finger sensor. A camera, placed $3 \mathrm{~m}$ from the participant, was used to capture videos images at 30fps, 960x720 resolution. b) Overview of the automated method used to recover the systolic and diastolic peaks from videos of a human face. 1) Facial landmarks are detected (using [9]) and the skin region of interest (ROI) segmented (excluding the region around the eyes), 2) spatial average of each color channel in the ROI over time calculated, 3) source signals, calculated using Independent Component Analysis (JADE implementation), are filtered, 4) the channel with the estimated strongest BVP signal selected and inverted (if necessary), 5) BVP signal interpolated to $256 \mathrm{~Hz}$ and systolic peaks detected, 6) second order derivative of the BVP waveform is calculated, interpolated to $256 \mathrm{~Hz}$, and used to locate the diastolic inflection point.

approach. The videos, recorded from a digital single lens reflex (DSLR) camera (at 30fps, 960x720 resolution), were exported in an uncompressed format. The physiological and video recordings were analyzed offline using custom software written in MATLAB (The Mathworks, Inc.). A facial landmark detector [9] was used to find the $\mathrm{x}$ - and $\mathrm{y}$ - coordinates of points on the face in each frame of the video. These were used to define a region of interest (ROI). We selected the full width between the outer eye corners (w) and a height twice the width (w above the eye corners to $\mathrm{w}$ below the eye corners) as a box encompassing the ROI. We exclude pixels within a region around the eyes which is of width $\mathrm{w}$ and height $\mathrm{w} / 2$. The average ROI size was less than $25 \%$ of the frame. A spatial average of the color channel pixel values within the resulting ROI were calculated for each frame to form raw signals $\mathrm{x}_{1}(\mathrm{t}), \mathrm{x}_{2}(\mathrm{t}), \ldots, \mathrm{x}_{N}(\mathrm{t})$ respectively (where $\mathrm{N}$ is the number of channels). The raw traces were detrended using a technique based on a smoothness priors approach [10]. The resulting signals were normalized by subtracting the mean and dividing by the standard deviation. We then apply Independent Component Analysis (ICA) (JADE implementation [11]) to recover source signals from the observations, maximizing the non-Gaussianity within the sources. Each of the source signals was band-pass filtered (Hamming window filter with low- and high-frequency cut-offs at 45 beats-per-minute (bpm) $(0.75 \mathrm{~Hz})$ and $270 \mathrm{bpm}(4.5 \mathrm{~Hz})$ respectively). To select the appropriate source signal we calculated the normalized fast Fourier transform (FFT) of each source and chose the source signal with the greatest frequency peak within the range 45$270 \mathrm{bpm}$. The source was also scaled by -1 if necessary using the approach in [7].

\section{B. Systolic and Diastolic Peak Detection}

Systolic peak times can be detected accurately from the BVP waveform as they are maxima within the signal. To do so the estimated BVP signal was interpolated with a cubic spline function at a sampling frequency of $256 \mathrm{~Hz}$. We used a custom peak detection algorithm with a moving time window of length $0.25 \mathrm{~s}$ to locate the peaks. To avoid artifacts (such as motion or ectopic beats) peaks intervals were filtered using the non-causal of variable threshold (NC-VT) algorithm [12] with a tolerance of $30 \%$. These parameters have been verified in prior work [6], [7].

However, the diastolic peaks (or inflections) are much more difficult to locate as they are not always maxima (and even if they are the systolic peaks are generally much bigger). In order to automatically find the diastolic peaks we first compute the second-order derivates of the BVP waveforms for both the contact and remote PPG measurements. These were computed by performing the MATLAB diff function twice on each of the signals. The outputs are smoothed using a three-point moving average filter. Figure 4 shows examples of the BVP waveforms and the second order derivatives for two of the subjects for a $5.5 \mathrm{~s}$ window. Figures 4(a) and (b) show an example with quite clear diastolic peaks and Figures 4(c) and (d) show another example with less obvious diastolic inflections.

In most cases the largest minima within the second order derivative correspond to the systolic peaks and the minima following these typically correspond to the diastolic peaks/inflections. We use the locations of the systolic peaks as detected above and perform peak detection on the inverted second order derivative waveform using a custom peak detection function in which a point is considered a peak if it is a local maxima and is greater than the preceding value by at least $1 \%$ of the total signal amplitude. The timing of the diastolic peak is located as the timing of the minima following the systolic peak in each pulse cycle. The systolic-diastolic peak-to-peak times (SD-PPT) are then calculated for each beat. As the SD-PPTs are relatively stationary (compared to IBIs) within each twominute session we class estimates that fall beyond one standard deviation from the mean as outliers and do not include these in our estimates of the final mean SD-PPT.

\section{EXPERIMENTS}

\section{A. Design}

Camera: The camera used for recording the video sequences was a digital single-lens reflex (DSLR) camera with a Zuiko 50mm lens. The lens power will have an impact on the accuracy of the measurements that can be made at a give distance. The camera has sensors for capturing five color bands which includes the typical three color band sensors (red, green and blue (RGB)) and also cyan and orange frequency band sensors (ROGCB). Specifically, the camera's CMOS sensor 
has pixels for detecting light in the orange and cyan frequency bands as well as pixels for detecting light in the red, green and blue bands. In the experiments described below we tested the performance of different color band combinations. The camera sensitivity profile and sensor layout can be found in [7] and information about the demosaicking in [13]. The changes to the CMOS sensor would not make such a sensor dramatically more expensive than a standard DSLR camera sensor.

Contact Measurements: Although there are no international standards for the measurement of PPG the most commonly used approach is to use a contact device with a dedicated light source and sensor [1]. Thus, for comparison we collected contact PPG measurements to validate the remote physiological measurements. The contact PPG signal was recorded using an FDA-approved sensor (Flexcomp Infiniti by Thought Technologies, Inc.) on the left index finger. Documentation for the contact sensor is available online [14]. As there is no ground truth measurement of PPG for comparison of measurements with the remote method we visually verified the systolic and diastolic peak detection for the contact signals.

\section{B. Protocol}

All experiments were conducted indoors with a varying amount of sunlight and indoor illumination. Participants were seated $3 \mathrm{~m}$ from the camera and the data were recorded on a laptop (Toshiba running Windows 7). Our experiments included 14 healthy participants of both genders (eight females), different ages (18-35) and skin color. During the experiment participants were asked to face the camera while the videos were recorded. Figure 2(a) shows the experimental setup. Two minute recordings of the participants were taken, the contact measurements and video sequences were synchronized. Our study was approved by the Institutional Review Board of the Massachusetts Institute of Technology.

Two recordings were taken for each participant, one at rest and one during a cognitive task. In all cases the cognitive task was performed after the measurements at rest:

Measurements at rest. Participants were asked to sit still, look toward the camera and relax. The video and contact recordings were captured for two minutes.

Measurements during cognitive task. Participants were asked to perform a mental arithmetic test (MAT) silently. Starting with the number 4000 they were required to subtract 7 , then subtract 7 again, and so on, as quickly as possible. The video and contact recordings were captured for two minutes. The participants started the task immediately after the recordings were started. Nearly all the participants reported this task to be significantly more stressful than the rest period.

\section{Quantifying Morphological Parameters}

In order to access the accuracy of the remote waveform morphology relative to the contact measurement we defined the following characteristics of the pulse wave. A summary of the parameters can be seen in Figure 1.

Inter-Beat Intervals (IBIs): Defined as the intervals between successive systolic peaks. a)
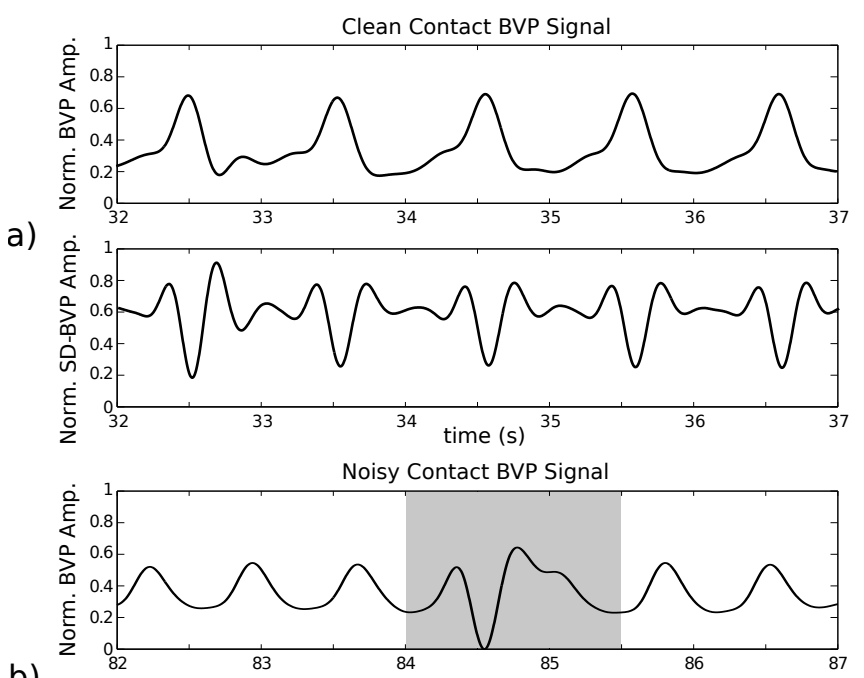

b)

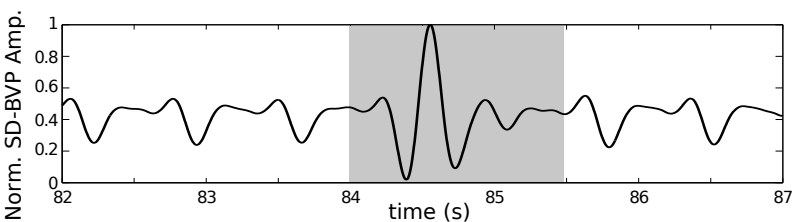

Fig. 3. Examples of a) clean and b) noisy contact sensor signals. The affected region is highlighted. For comparison with the remote method it was important that the systolic and diastolic peaks in the contact measures could be visually verified since there is no clinically approved ground-truth method for automatic detection of PPG systolic or diastolic peaks.

Systolic-Diastolic Peak-to-Peak Times (SD-PPTs): Defined as the time between the systolic and diastolic peak for each pulse wave.

\section{RESULTS AND DisCUSSION}

Using the data collected in the experiments described in Section III-B we tested the performance of our approach for automatically detecting systolic and diastolic peaks. For three participants the contact sensor measurements were noisy and had a number of unverifiable systolic and diastolic peaks. If we could not visually verify the peaks within the waveforms we did not use the sessions for the comparison with the camera method here. This highlights some of the challenges associated with contact sensor measurements. Figure 3 shows two examples of noisy contact measurements. Notice that the shape of the waveforms changes significantly in the affected region and the systolic and diastolic peaks/inflections cannot be verified. The noise within the signals is most likely to be due to movement of the fingers.

\section{A. Morphology Statistics}

Table I shows the mean and standard deviations of the IBIs and the SD-PPTs, measured using the contact sensor, for the rest and stress cases. As we would expect the standard deviation of the IBIs with each session is much greater than the standard deviation of the SD-PPTs. We would not expect the SD-PPTs to vary a lot within a session or for an individual between tasks as this is a result of the time taken for the reflected pulse wave to return from the lower 
(a)
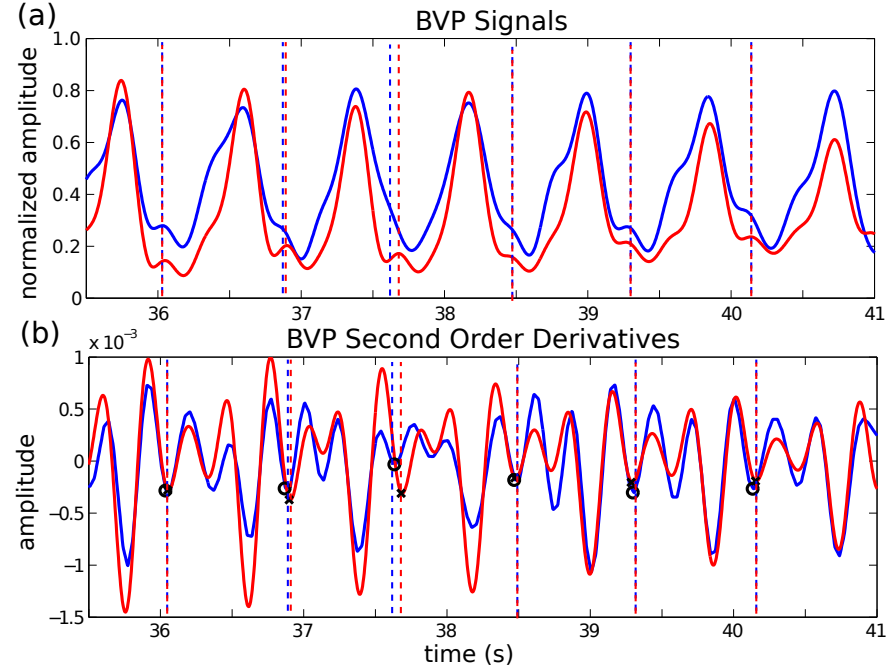

(c)

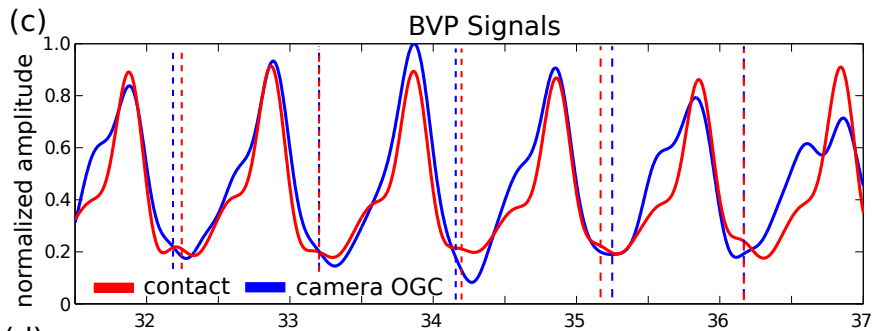

(d)

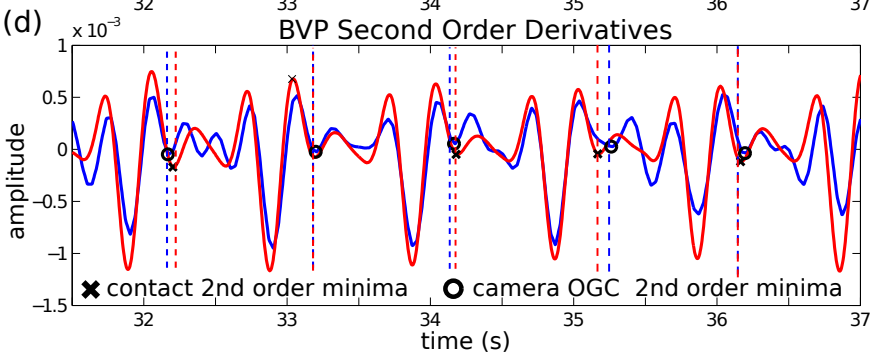

Fig. 4. BVP and BVP second order derivatives measured using a contact finger sensor (red) and a digital camera (blue). a) BVP waveforms with more prominent dicrotic notch and b) corresponding second order derivatives and estimation of diastolic peak locations. c) BVP waveforms with less prominent dicrotic notch and d) corresponding second order derivatives and estimation of diastolic peak locations.

TABLE I

MEAN (AND STANDARD DEVIATION) OF IBIS AND SD-PPTS FOR EACH OF THE 11 PARTICIPANTS DURING THE REST AND STRESS TASKS.

\begin{tabular}{ccccc}
\hline \hline & \multicolumn{2}{c}{ IBIs (s) } & \multicolumn{2}{c}{ SD-PPTs (s) } \\
& Rest & Stress & Rest & Stress \\
\hline P1 & $0.99(0.038)$ & $0.95(0.047)$ & $0.29(0.006)$ & $0.28(0.007)$ \\
P2 & $0.97(0.040)$ & $0.99(0.049)$ & $0.30(0.009)$ & $0.32(0.027)$ \\
P3 & $0.84(0.045)$ & $0.76(0.060)$ & $0.29(0.007)$ & $0.30(0.024)$ \\
P4 & $0.85(0.064)$ & $0.89(0.061)$ & $0.32(0.015)$ & $0.32(0.013)$ \\
P5 & $0.83(0.037)$ & $0.79(0.031)$ & $0.31(0.010)$ & $0.30(0.008)$ \\
P6 & $0.91(0.059)$ & $0.87(0.055)$ & $0.33(0.010)$ & $0.32(0.012)$ \\
P7 & $0.90(0.058)$ & $0.87(0.036)$ & $0.29(0.009)$ & $0.31(0.007)$ \\
P8 & $1.00(0.070)$ & $0.75(0.046)$ & $0.34(0.011)$ & $0.28(0.004)$ \\
P9 & $0.75(0.046)$ & $0.75(0.046)$ & $0.28(0.004)$ & $0.28(0.005)$ \\
P10 & $0.91(0.046)$ & $0.81(0.046)$ & $0.29(0.025)$ & $0.30(0.010)$ \\
P11 & $0.82(0.057)$ & $0.85(0.045)$ & $0.32(0.009)$ & $0.31(0.006)$ \\
\hline \hline Mean & $0.89(0.051)$ & $0.84(0.047)$ & $0.31(0.011)$ & $0.30(0.011)$
\end{tabular}

limbs and is not controlled by the autonomic nervous system (ANS). However, there is variation in mean SD-PPT between individuals, as much as $60 \mathrm{~ms}$. The mean standard deviation of the IBIs was close to $50 \mathrm{~ms}$ for both tasks and there was a significant difference in the relative high and low frequency power components in the HRV frequency spectra between tasks. The HRV frequency spectrum is the frequency spectrum of the IBI time series. The low frequency (LF) and high frequency (HF) powers of the HRV were calculated as the area under the PSD curve corresponding to 0.04-0.15 and 0.15$0.4 \mathrm{~Hz}$ respectively. $\mathrm{LF} / \mathrm{HF}$ power ratio was significantly higher during the stress task (1.66) than the rest task (0.84). This is expected as HRV is influenced by both the sympathetic and parasympathetic branches of the ANS.

\section{B. Validation of Remote Measurement of Morphology}

Excluding the data from the noisy contact measurements leaves 22 two minute sessions from 11 participants. Figure 4 shows samples of the BVP signals calculated from the face using the digital camera (blue lines) and the second order derivatives of these signals. There are clear minima in the second order derivatives that correspond to the diastolic peaks (or inflections). The maxima immediately preceding these corresponds to the dicrotic notch. In some cases the inflections are visible in the BVP waveform. However, in other cases the inflections are only obvious by looking at the second order derivative (see Figure 4(c) and (d) in particular). In some cases (see Figure 4(d)) the second order derivatives have additional waves - perhaps due to artifacts within the detected BVP waveform.

Although we would not expect the timings of the diastolic peaks to be identical at the finger and on the face (due to the reflected wave being different at different parts of the body) it is likely that there would be a high degree of agreement. Figure 4 compares the BVP waveforms and second order derivatives for the contact finger measurements (red lines) and the camera measurements (blue lines). The timings of peaks within the contact and remotely measured BVP waveforms and derivatives are very similar. The diastolic and systolic peak heights are similar but not always identical, this may be in part due to the difference in the magnitude of the waves observed at different parts of the body.

The top row of Figure 5 shows the plots of the systolic peak intervals (the same as the inter-beat intervals (IBIs)) measured using the contact sensor and the camera method for three subjects. The bottom row of Figure 5 shows the SD-PPTs measured using the contact sensor and the camera method for the same three subjects. We compare the measurements using the camera green channel alone and the OGC channel combination. For these examples we can see extremely close agreement between the measurements from the camera and the contact sensor over a two minute period using the OGC channel combination. Figures 5 (a) and (b) shows an example in which the OGC channel combination performed well, using the green channel alone the measurements are less accurate. Figure 5(c) shows an example in which the green and OGC channels both performed quite well in estimating the IBIs but poorly in estimating the SD-PPTs. 

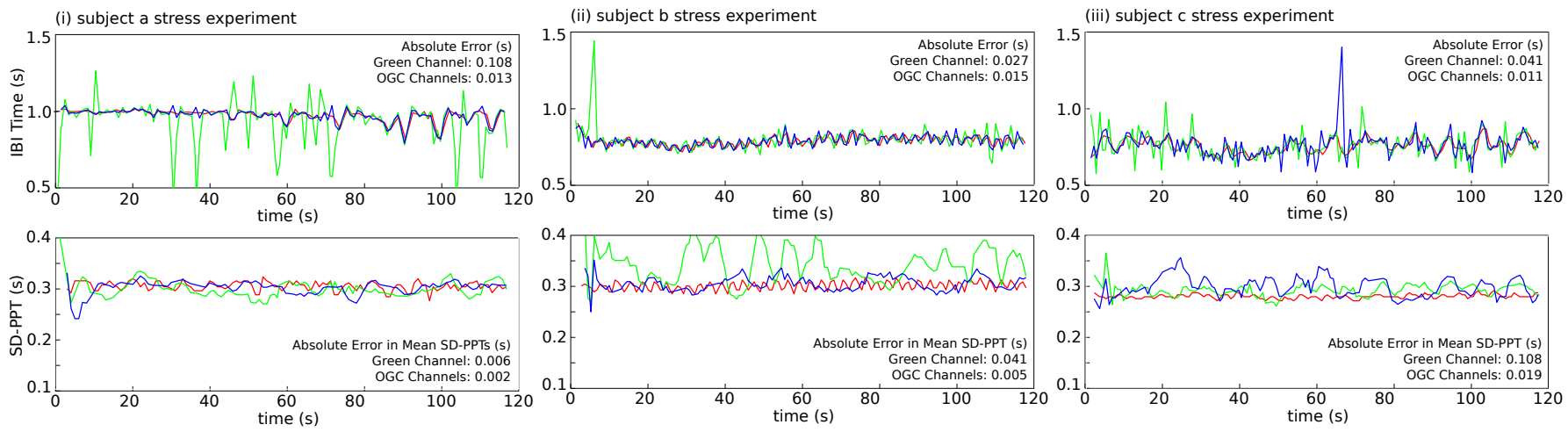

Fig. 5. Top row) Systolic peak intervals (inter-beat intervals) measured using a contact sensor (red), the camera green channel (green) and the camera OGC combination (blue) for three subjects over two minutes. Bottom row) systolic-diastolic peak-to-peak times (SD-PPT) measured using a contact sensor (red), the camera green channel (green) and the camera OGC combination (blue) for three subjects over two minutes. a) An example in which the OGC combination performed very well and much better than the green channel, especially in IBI estimation, b) An example in which the OGC combination performed very well and much better than the green channel, especially in SD-PPT estimation, c) an example where both the green and OGC channel camera methods performed relatively poorly in the estimation of SD-PPTs.

TABLE II

THE MEAN \% ABSOLUTE ERROR AND ABSOLUTE ERROR BETWEEN THE CONTACT AND REMOTELY MEASURED MORPHOLOGY PARAMETERS FOR ALL 22 SESSIONS FROM 11 PARTICIPANTS.

* - SIGNIFICANTLY LOWER ERROR THAN RGB $(p<0.05)$

** - SIGNIFICANTLY LOWER ERROR THAN BOTH G AND RGB $(p<0.05)$.

\begin{tabular}{cccccc}
\hline \hline & & \multicolumn{4}{c}{ Channels } \\
& & $\mathrm{G}$ & $\mathrm{RGB}$ & $\mathrm{OGC}$ & $\mathrm{ROGCB}$ \\
\hline \multirow{3}{*}{ IBIs } & \% Ab.Error & $5.12^{*}$ & 8.72 & $3.10^{* *}$ & $3.10^{* *}$ \\
& Ab.Error (s) & $0.045^{*}$ & 0.076 & $0.026^{* *}$ & $0.026^{* *}$ \\
Mean SD-PPT & \% Ab.Error & 5.47 & 5.79 & 4.11 & 4.13 \\
& Ab.Error (s) & 0.016 & 0.017 & 0.012 & 0.013
\end{tabular}

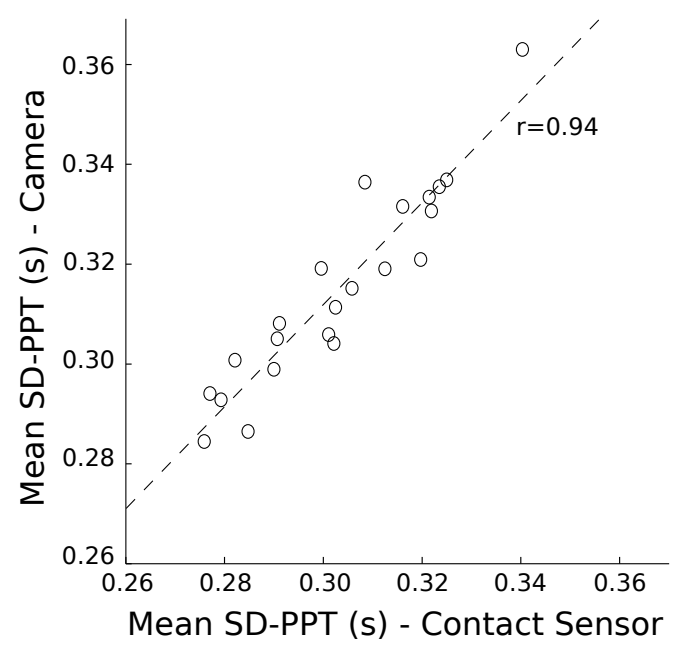

Fig. 6. Mean SD-PPT measured using the remote method and the camera for the 11 participants and 22 sessions (correlation: $0.94, p<0.001$ ).

Table II shows the mean percentage absolute error and the mean absolute error between the contact and remotely measured systolic peak intervals for all 22 sessions. Table II also shows the percentage absolute error and mean absolute error between the contact and remotely measured mean systolic-diastolic peak-to-peak times. We compare the results calculated using just the green channel $(G)$, the red, green and blue channels (RGB), the orange, green and cyan channels
(OGC) and all channels (ROGCB). The absolute error between the contact and remotely measured IBIs using OGC is $0.026 \mathrm{~s}$ (this represents only 3\% error). The errors between the contact and remotely measured mean SD-PPTs are also small, $0.012 \mathrm{~s}$ absolute error, using OGC channels (this represents $4 \%$ error in SD-PPT timing). The accurate estimation of systolic and diastolic peak locations is particularly good considering that the sampling rate of the video was only $30 \mathrm{~Hz}$. The SD-PPTs are much more challenging to calculate than the IBIs as the diastolic peaks/inflections are more subtle - often a peak is not present. These results support those reported previously in [7]; the OGC channel combination significantly outperforms the green and RGB channels for measurement of physiological parameters (here the significances were computed using two-sample Kolmogorov-Smirnov tests). The OGC channels performs equivalently to the ROGCB combination. Again, this suggests there is no added benefit of the red and blue channels.

Figure 6 shows the scatter plot of the mean SD-PPT for each of the 22 sessions measured using the contact sensor and the camera method. There is a strong correlation ( $\rho=$ 0.94, $p<0.001)$. The SD-PPTs measured using the camera are typically slightly longer than those measured using the finger sensor.

\section{Comparison of Accuracy During Rest and Cognitive Task}

Table III shows the performance of the method (using the OGC channels) during the rest and the stress task separately. The results show that the performance was comparable for both tasks. The error in IBIs for the rest task was $3.11 \%$ and for the cognitive stress task was $3.10 \%$. The error in mean SDPPT for the rest task was $4.60 \%$ and for the cognitive stress task was $3.62 \%$. As the mean SD-PPTs are not significantly different during each of the tasks it is reasonable that the performance of the automatic detection would be similar. The IBI measurements were accurate enough to measure the difference in IBI intervals between the two tasks. 
TABLE III

THE MEAN \% ABSOLUTE ERROR AND ABSOLUTE ERROR BETWEEN CONTACT AND REMOTE MEASURES OF MORPHOLOGY PARAMETERS FOR ALL 11 PARTICIPANTS DURING THE REST AND COGNITIVE STRESS TASKS.

\begin{tabular}{cccc}
\hline \hline & & \multicolumn{2}{c}{ Task } \\
& & Rest & Cog. Stress \\
\hline \multirow{3}{*}{ IBIs } & \% Ab.Error & 3.11 & 3.10 \\
& Ab.Error (s) & 0.027 & 0.025 \\
Mean SD-PPT & $\%$ Ab.Error & 4.60 & 3.62 \\
& Ab.Error (s) & 0.014 & 0.011
\end{tabular}

\section{CONCLUSIONS AND FutURE WORK}

We have presented a new automated method for detection of the systolic and diastolic peaks of a PPG waveform captured remotely using a digital camera. Thus we can automatically capture the peak-to-peak times which are useful in measuring aspects of cardiac health. We have shown that the second order derivative of the camera PPG waveform reveals the diastolic peak (or inflection) even if it does not appear to be visible in the original PPG waveform.

We compare the measurements of diastolic and systolic peak times measured remotely from the face with those measured from a contact finger tip sensor. The results show that there is high agreement in the peak-to-peak time and the inter-beat intervals. The best performance was obtained with a combination of green, orange and cyan color bands from the camera. The correlation between the mean SD-PPT measured from the contact sensor and the remote method was $0.94(p<0.01)$. The accuracy of the predictions did not vary significantly during a restful and cognitively stressing task. Using a combination of the orange, green and cyan channels yields significantly better $(p<0.01)$ results than using just the green signal or the traditional RGB combination.

The waveform morphology captured using ambient light and a remote camera is susceptible to motion artifacts. Although in the data we present here the participants were free to move, they were seated and not talking - therefore relatively stationary. Future work should consider how the estimates of peak times are degraded in the presence of rigid head motions. In addition, we plan to apply the technique to videos of individuals from a broader range of ages in order to investigate whether the effect of aging on the waveform morphology can be recovered using these techniques.

Defining the operational envelope of remote PPG measurement using digital cameras is important and there will be a trade-off between the camera lens power and distance at which measurements can be made with a similar accuracy. All analysis was performed offline - we leave a real-time implementation for future work.

Morphological parameters from the PPG could have the potential to predict arterial stiffness (AS) which is an early sign of atherosclerosis (hardening of the arteries) [15]. Properties of the waveform can be used as a measure of small and medium arterial stiffness due to the relationship between arterial elasticity and the reflection of the PPG signal. Also, with age the distance between the diastolic point and the systolic point decreases. Future work will investigate whether the relative amplitude of the systolic and diastolic peaks can be accurately recovered using this method.

\section{ACKNOWLEDGEMENTS}

This work was funded by the MIT Media Member consortium. Daniel McDuff was supported by an NEC fellowship.

\section{REFERENCES}

[1] J. Allen, "Photoplethysmography and its application in clinical physiological measurement," Physiological measurement, vol. 28, no. 3, p. R1, 2007.

[2] J. Allen, C. P. Oates, T. A. Lees, and A. Murray, "Photoplethysmography detection of lower limb peripheral arterial occlusive disease: a comparison of pulse timing, amplitude and shape characteristics," Physiological measurement, vol. 26, no. 5, p. 811, 2005.

[3] A. M. Brumfield and M. E. Andrew, "Digital pulse contour analysis: investigating age-dependent indices of arterial compliance," Physiological measurement, vol. 26, no. 5, p. 599, 2005.

[4] K. Takazawa, N. Tanaka, M. Fujita, O. Matsuoka, T. Saiki, M. Aikawa, S. Tamura, and C. Ibukiyama, "Assessment of vasoactive agents and vascular aging by the second derivative of photoplethysmogram waveform," Hypertension, vol. 32, no. 2, pp. 365-370, 1998.

[5] M.-Z. Poh, D. J. McDuff, and R. W. Picard, "Non-contact, automated cardiac pulse measurements using video imaging and blind source separation," Optics Express, vol. 18, no. 10, pp. 10762-10 774, 2010.

[6] _ - "Advancements in noncontact, multiparameter physiological measurements using a webcam," Biomedical Engineering, IEEE Transactions on, vol. 58, no. 1, pp. 7-11, 2011.

[7] D. McDuff, S. Gontarek, and R. Picard, "Improvements in remote cardio-pulmonary measurement using a five band digital camera," Biomedical Engineering, IEEE Transactions on, vol. 61, no. 9, 2014.

[8] Y. Sun, S. Hu, V. Azorin-Peris, S. Greenwald, J. Chambers, and Y. Zhu, "Motion-compensated noncontact imaging photoplethysmography to monitor cardiorespiratory status during exercise," Journal of Biomedical Optics, vol. 16, no. 7, pp. 077 010-077 010, 2011.

[9] B. Martinez, M. F. Valstar, X. Binefa, and M. Pantic, "Local evidence aggregation for regression-based facial point detection," IEEE Transactions on Pattern Analysis and Machine Intelligence, vol. 35, no. 5, pp. 1149-1163, 2013.

[10] M. P. Tarvainen, P. O. Ranta-aho, and P. A. Karjalainen, "An advanced detrending method with application to hrv analysis," Biomedical Engineering, IEEE Transactions on, vol. 49, no. 2, pp. 172-175, 2002.

[11] J.-F. Cardoso and A. Souloumiac, "Blind beamforming for non-gaussian signals," in IEE Proceedings F (Radar and Signal Processing), vol. 140, no. 6. IET, 1993, pp. 362-370.

[12] J. Vila, F. Palacios, J. Presedo, M. Fernández-Delgado, P. Felix, and S. Barro, "Time-frequency analysis of heart-rate variability," Engineering in Medicine and Biology Magazine, IEEE, vol. 16, no. 5, pp. 119$126,1997$.

[13] Y. Monno, M. Tanaka, and M. Okutomi, "Multispectral demosaicking using guided filter," in IS\&T/SPIE Electronic Imaging. International Society for Optics and Photonics, 2012, pp. 82990O-82990O.

[14] T. Technology. Flexcomp infiniti hardware manual. [Online]. Available: http://www.thoughttechnology.com/pdf/manuals/SA7560\%20rev.\%204 \%20FlexComp\%20Infiniti\%20User\%20Manual\%20(2).pdf

[15] J. J. Oliver and D. J. Webb, "Noninvasive assessment of arterial stiffness and risk of atherosclerotic events," Arteriosclerosis, Thrombosis, and Vascular Biology, vol. 23, no. 4, pp. 554-566, 2003.

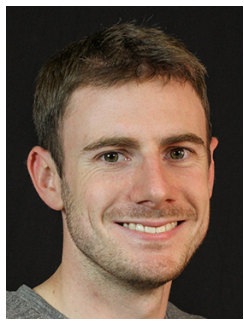

Daniel McDuff (S'09) received the bachelor's degree, with first-class honors and master's degree in engineering from Cambridge University. He received the $\mathrm{PhD}$ degree in Media Arts and Sciences from MIT while working at the MIT Media Lab. He is interested in computer vision and machine learning to enable the automated recognition of affect. He is also interested in technology for remote measurement of physiology. 


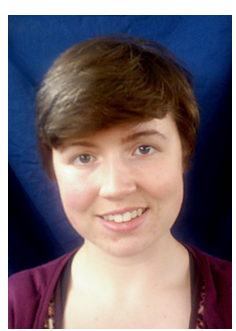

Sarah Gontarek is an electrical engineering and computer science student at MIT, and a political science student at Wellesley College. She is interested in signal processing, neuroscience, and machine learning. She enjoys work in the Affective Computing Group in the Media Lab, because she is especially interested in biomedical applications for common digital communications technology.

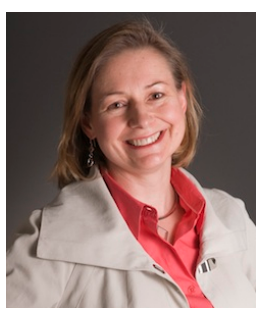

Rosalind W. Picard (M'81 - SM'00 - F'05) received the $\mathrm{ScD}$ degree in electrical engineering and computer science from MIT. She is a professor of Media Arts and Sciences at the MIT Media Lab, founder and director of the Affective Computing Group at the MIT Media Lab. She is also a co-founder of Affectiva, Inc. and Empatica, Inc. Her current research interests focus on the development of technology to help people comfortably and respectfully measure and communicate affective information, as well as on the development of models of affect that improve decision-making and learning. She is a fellow of the IEEE and member of the IEEE Computer Society. 